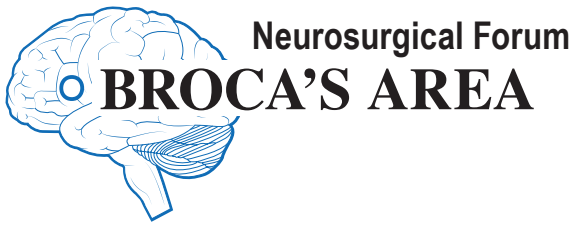

\section{Biopsy for diffuse intrinsic pontine glioma: a reappraisal}

Mark W. Kieran, MD, PhD, ${ }^{1}$ Liliana C. Goumnerova, MD, ${ }^{2}$ Michael Prados, MD, ${ }^{3}$ and Nalin Gupta, MD, PhD ${ }^{3}$

1Dana Farber Cancer Institute, Boston, MA

2Boston Children's Hospital, Boston, MA

${ }^{3}$ University of California, San Francisco, CA

$\mathrm{D}$ IFFUSE intrinsic pontine gliomas (DIPGs) are regionally specific gliomas that arise in a relatively narrow age range in children. As opposed to a pontine location, tumors that arise in the medulla or midbrain are often WHO Grade I or II tumors and have a markedly different prognosis than DIPGs. The characteristic location as well as the presence of extensive infiltration in the ventral pons means that resection is not possible. The overall lack of treatment options, and the resulting dismal prognosis associated with a diagnosis of DIPG has historically led to a sense of nihilism among treating physicians. The biological behavior of these tumors has long been thought to best fit with adult WHO Grade IV astrocytoma, and it was also assumed that the molecular features would be similar to those observed in other locations in the brain. These assumptions led to the use of many experimental agents that are used in the context of adult clinical trials for WHO Grade IV astrocytoma. However, this did not result in any meaningful improvement in survival. ${ }^{7}$

The recent analysis of tissue obtained from a variety of sources by different groups has shown conclusively that primary brain tumors in children have distinct features when compared to adult gliomas..$^{1,4,12,15}$ Furthermore, the majority of DIPGs have a particularly unique molecular signature, that of mutations in the histone genes, which leads to changes in gene expression that are believed to account for their oncogenesis. ${ }^{3,9,14}$ In addition, pharmacological alterations in histone methylation appear to result in inhibition of tumor growth, at least in preclinical studies. ${ }^{5,6}$

The biological advances as well as the surgical techniques that have been present for the last 10-20 years have allowed biopsies to be performed with acceptable morbidity. ${ }^{2,11}$ In the series published to date, the majority of complications were transient cranial neuropathies, although more serious ones have been reported. Although the potential for surgical morbidity cannot be ignored or minimized, there is a path forward in terms of the molecular and biological characterization of DIPGs that can be used to further refine therapeutic decisions ${ }^{10}$ : specifically, biopsy-directed clinical trials that use tissue to assign patients to rational therapeutic arms are under way.

Recently opened trials using advanced sequencing techniques to identify molecular alterations within specific pathways for a patient's tumor have allowed therapy to be directed against those targets-an example of personalized medicine (clinicaltrial.gov NCT01182350 and NCT02233049). Of course, it is not clear whether this approach casts too broad a net, or whether other factors such as inadequate drug delivery will lead to failure. However, within the broader scope of precision medicine, and perhaps uniquely for children with rare cancers, continued development of rationally designed clinical trials is needed. In an era of rapid acquisition of RNA and DNA deep sequencing information in individual cases, "rational" means using tumor-derived molecular information wisely. Strategies that use genomic data within individual patients are ongoing in the treatment of most cancers, including adult glioma and now, hopefully, in children within all subsets of CNS tumors. Tumor tissue is a requirement to achieve these goals.

Resection and/or biopsy of tumors located in many other eloquent regions of the brain such as the thalamus, insula, perisylvian location, and in the spinal cord are considered the standard of care. Whereas most neurosurgeons would consider biopsy of other areas of the brain acceptable, this perspective is generally not applied to patients 
with DIPG. At this point, the ability to identify biological subtypes with rapid genomic profiling linked to potentially actionable therapeutics, and a clear understanding of surgical risk, provide a justification for considering and not fearing the option of surgical biopsy. For most centers, this means that surgical biopsy in the context of an approved and rational clinical trial with experienced neurosurgeons should be viewed as the standard of care for this disease. ${ }^{8,13}$

\section{References}

1. Buczkowicz P, Hoeman C, Rakopoulos P, Pajovic S, Letourneau L, Dzamba M, et al: Genomic analysis of diffuse intrinsic pontine gliomas identifies three molecular subgroups and recurrent activating ACVR1 mutations. Nat Genet 46:451456, 2014

2. Cage TA, Samagh SP, Mueller S, Nicolaides T, Haas-Kogan D, Prados M, et al: Feasibility, safety, and indications for surgical biopsy of intrinsic brainstem tumors in children. Childs Nerv Syst 29:1313-1319, 2013

3. Chan KM, Fang D, Gan H, Hashizume R, Yu C, Schroeder $\mathrm{M}$, et al: The histone $\mathrm{H} 3.3 \mathrm{~K} 27 \mathrm{M}$ mutation in pediatric glioma reprograms $\mathrm{H} 3 \mathrm{~K} 27$ methylation and gene expression. Genes Dev 27:985-990, 2013

4. Fontebasso AM, Papillon-Cavanagh S, Schwartzentruber J, Nikbakht H, Gerges N, Fiset PO, et al: Recurrent somatic mutations in ACVR1 in pediatric midline high-grade astrocytoma. Nat Genet 46:462-466, 2014

5. Grasso CS, Tang Y, Truffaux N, Berlow NE, Liu L, Debily MA, et al: Functionally defined therapeutic targets in diffuse intrinsic pontine glioma. Nat Med 21:555-559, 2015 (Erratum in Nat Med 21:827, 2015)

6. Hashizume R, Andor N, Ihara Y, Lerner R, Gan H, Chen X, et al: Pharmacologic inhibition of histone demethylation as a therapy for pediatric brainstem glioma. Nat Med 20:13941396, 2014
7. Jansen MH, van Vuurden DG, Vandertop WP, Kaspers GJ: Diffuse intrinsic pontine gliomas: a systematic update on clinical trials and biology. Cancer Treat $\operatorname{Rev} 38: 27-35$, 2012

8. Kieran MW: Time to rethink the unthinkable: upfront biopsy of children with newly diagnosed diffuse intrinsic pontine glioma (DIPPG). Pediatr Blood Cancer 62:3-4, 2015

9. Paugh BS, Broniscer A, Qu C, Miller CP, Zhang J, Tatevossian RG, et al: Genome-wide analyses identify recurrent amplifications of receptor tyrosine kinases and cell-cycle regulatory genes in diffuse intrinsic pontine glioma. J Clin Oncol 29:3999-4006, 2011

10. Robison NJ, Kieran MW: Diffuse intrinsic pontine glioma: a reassessment. J Neurooncol 119:7-15, 2014

11. Roujeau T, Machado G, Garnett MR, Miquel C, Puget S, Geoerger B, et al: Stereotactic biopsy of diffuse pontine lesions in children. J Neurosurg 107 (1 Suppl):1-4, 2007

12. Taylor KR, Mackay A, Truffaux N, Butterfield YS, Morozova O, Philippe C, et al: Recurrent activating ACVR1 mutations in diffuse intrinsic pontine glioma. Nat Genet 46:457-461, 2014

13. Walker DA, Liu J, Kieran M, Jabado N, Picton S, Packer R, et al: A multi-disciplinary consensus statement concerning surgical approaches to low-grade, high-grade astrocytomas and diffuse intrinsic pontine gliomas in childhood (CPN Paris 2011) using the Delphi method. Neuro Oncol 15:462468, 2013

14. Wu G, Broniscer A, McEachron TA, Lu C, Paugh BS, Becksfort J, et al: Somatic histone $\mathrm{H} 3$ alterations in pediatric diffuse intrinsic pontine gliomas and non-brainstem glioblastomas. Nat Genet 44:251-253, 2012

15. Wu G, Diaz AK, Paugh BS, Rankin SL, Ju B, Li Y, et al: The genomic landscape of diffuse intrinsic pontine glioma and pediatric non-brainstem high-grade glioma. Nat Genet 46:444-450, 2014

\section{Disclosures}

The authors report no conflict of interest. 